\title{
ANALYSIS OF ACOUSTIC ATTENUATION SPECTRA OF LIPON GLASSES
}

The acoustic attenuation spectra which reflect the basic features of the relaxation and transport processes of the mobile ions of investigated phosphate and oxynitride phosphate glasses are analyzed. Suitable theoretical models and mathematical fit of acoustic spectra are used for characterization of the ionic hopping motion, relaxation processes and transport mechanisms connected with the mobility of conductive ions. Results from acoustic measurements are compared with results obtained from electrical conductivity measurements.

\section{Introduction}

One of the most important requirements of the electrolyte materials in battery application is high ionic conductivity. Using solid electrolytes in rechargeable batteries can provide numerous advantages such as thermal and mechanical stability, high reliability, longer active life, the possibility of miniaturization through thin-film production.

Lithium-ion conductive glasses have been widely studied due to their potential application as solid-state amorphous electrolytes in secondary batteries [1]. Lithium metal can be used as the anode material in lithium batteries and it allows higher energy and power densities than when graphitized anodes are used as anode materials. It was found that the amorphous character of the material and the presence of nitrogen increase the conductivity with respect to that of the crystalline compound. The increase in conductivity is supposed to be related to the formation of $\mathrm{P}-\mathrm{N}$ bonds, which replace $\mathrm{P}-\mathrm{O}$ ones leading to a more reticulated anionic network [2].

The optical and electrochemical properties of lithium phosphorous oxynitride (LiPON) thin films have been studied and successfully applied in lithium microbatteries [3]. LiPON exhibits a single, $\mathrm{Li}^{+}$-ion conducting phase with an average conductivity of $(2.3 \pm 0.7) \times 10^{-6} \mathrm{~S} / \mathrm{cm}$ at $25^{\circ} \mathrm{C}$ and an average activation energy of $E_{a}=(0.55 \pm 0.02) \mathrm{eV}[3]$.

The acoustic attenuation measurement seems to be a useful technique for nondestructive investigation of transport mechanisms in conductive glasses and compared to the electrical ones it has even some advantages as high sensitivity, the absence of contact phenomena and so on $[4,5]$. Acoustical measurements made over a wide range of frequencies and temperatures can characterize different relaxation processes according to corresponding transport mechanisms due to the strong acousto-ionic interaction. In glassy electrolytes, the mobile ions encounter different kinds of site and ionic hopping motion and relaxation processes connected with charge mobility so that modified jump relaxation model $[6,7]$ can be used for transport mechanisms description.

In this contribution we present first results from acoustic investigation of lithium phosphate and oxynitride phosphate glasses with different $\mathrm{Li} / \mathrm{P}$ ratios, first with compositions $57.5 \mathrm{Li}_{2} \mathrm{O} \cdot 42.5 \mathrm{P}_{2} \mathrm{O}_{5}$ and second prepared through a thermal treatment under ammonia atmosphere of the sample with initial composition $55 \mathrm{Li}_{2} \mathrm{O} \cdot 45 \mathrm{P}_{2} \mathrm{O}_{5}$. A theoretical model is suggested for the description of experimental results obtained from acoustic spectra of the glasses prepared in the system $\mathrm{Li}_{1.35} \mathrm{PO}_{3.18}$ and $\mathrm{Li}_{1.22} \mathrm{PO}_{2.80} \mathrm{~N}_{0.21}$ with the purpose to study ion relaxation processes and transport mechanisms.

\section{Experiment}

\subsection{Characterisation of the glasses}

Lithium phosphate glasses with composition $\mathrm{xLi}_{2} \mathrm{O} \cdot(1-\mathrm{x}) \mathrm{P}_{2} \mathrm{O}_{5}$ $(\mathrm{x}=55,57.5 \mathrm{~mol} \%)$, were obtained by conventional melt-quenching technique. Stoichiometric amounts of reagent grade raw materials $\mathrm{Li}_{2} \mathrm{CO}_{3}$ (Aldrich, $99 \%$ ) and $\left(\mathrm{NH}_{4}\right)_{2} \mathrm{HPO}_{4}$ (Merck, $99 \%$ ) were weighed and mixed. The batches were calcined in porcelain crucibles held in an electric furnace up to $450{ }^{\circ} \mathrm{C}$, and then melted in a gas furnace (propane/air) during $1 \mathrm{~h}$ at temperatures ranging from $800{ }^{\circ} \mathrm{C}$ to $1000{ }^{\circ} \mathrm{C}$ depending on composition. The compositions of the glasses are given by their molecular formula as $\mathrm{Li}_{1.22} \mathrm{PO}_{3.11}$ and $\mathrm{Li}_{1.35} \mathrm{PO}_{3.18}$ for $\mathrm{Li}_{2} \mathrm{O}$ contents of 55 and $57.5 \mathrm{~mol} \%$, respectively [2].

Oxynitride lithium phosphate glasses were obtained through ammonolysis of the base glasses in an $\mathrm{Al}_{2} \mathrm{O}_{3}$ gas-tight tube furnace

\footnotetext{
* Peter Hockicko ${ }^{1}$, Francisco Munoz ${ }^{2}$, Peter Bury ${ }^{1}$, Peter Sidor ${ }^{1}$

${ }^{1}$ Department of Physics, Faculty of Electrical Engineering, University of Zilina, Slovakia

${ }^{2}$ Insituto do Ceramica y Vidrio (CSIC), Madrid, Spain
} 
at temperatures from $600{ }^{\circ} \mathrm{C}$ to $750{ }^{\circ} \mathrm{C}$ and treatment times of $3 \mathrm{~h}$. Base glasses were placed in graphite moulds acting as individual "crucibles" of $2 \mathrm{~cm}$ in diameter and $5 \mathrm{~mm}$ deep. The furnace was heated up to the treatment temperature at a constant heating rate of $10 \mathrm{~K} \mathrm{~min}^{-1}$ under $\mathrm{N}_{2}$ flow. In the present work only two glass compositions of prepared set of samples [2] are studied by acoustic spectroscopy: $\mathrm{Li}_{1.35} \mathrm{PO}_{3.18}(\mathrm{C})$ and $\mathrm{Li}_{1.22} \mathrm{PO}_{2.80} \mathrm{~N}_{0.21}$ (BN).

Electrical conductivity measurements were performed by Electrochemical Impedance Spectroscopy (EIS) in a Solartron SI1260 impedance analyzer, in the frequency range from $10 \mathrm{~Hz}$ to $10 \mathrm{MHz}$ at temperatures between 40 and $200{ }^{\circ} \mathrm{C}$ [2]. The samples were cut into discs of 1-2 $\mathrm{mm}$ in thickness and $10 \mathrm{~mm}$ in diameter and gold electrodes were sputtered on both faces as contacts for electrical measurements.

\subsection{Acoustic measurements}

The longitudinal acoustic waves of frequency 13, 17 and 29 $\mathrm{MHz}$ were generated by Pulse Modulator and Receiver - MATEC 7700 and $\mathrm{LiNbO}_{3}$ transducer acoustically bonded to the quartz rod buffer. Acoustic measurements were realized at the temperature range of $290-530 \mathrm{~K}$ at a heating rate of $0.5 \mathrm{~K} / \mathrm{min}$ on the same samples that were used previously for electrical measurements. The quartz buffer was used to separate the acoustic signal from thin sample (Fig. 1). The samples for acoustical attenuation measurements were cylindrical in shape (thickness $\approx 1.8 \mathrm{~mm}$ and $8.7 \mathrm{~mm}$ in diameter) and end faces were polished to be flat and parallel.

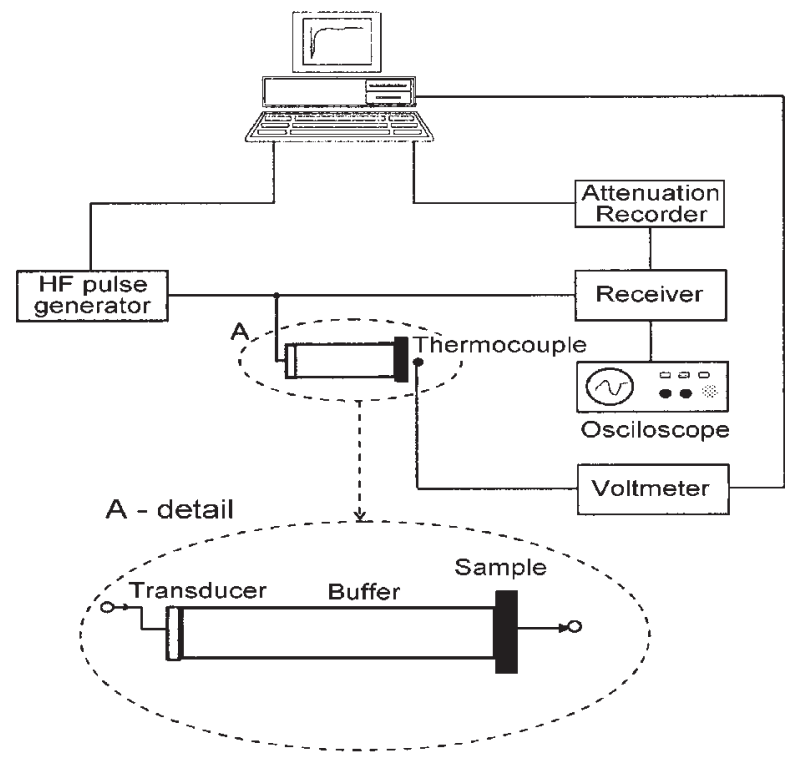

Fig. 1 Experimental arrangement for acoustic attenuation measurement

\section{Theoretical principles}

In a dilute system containing a low concentration of mobile ions the acoustic attenuation spectrum may be described as a Debye-like, single relaxation time process in which the individual ion hops occur independently of each other. In such cases, the attenuation $\alpha$ for a wave of angular frequency $\omega$ takes the form

$$
\alpha=\Delta\left(\frac{\omega^{2} \tau}{1+\omega^{2} \tau^{2}}\right),
$$

where the parameter $\Delta$ is the relaxation strength and it determines the magnitude of the attenuation peak [8].

The term in the equation (1) in the round brackets describes a Debye peak. The acoustic attenuation will exhibit a maximum when the condition $\omega \tau$ is equal to 1 and

$$
\tau=\tau_{0} \exp \left(E_{a} / k_{B} T_{\text {peak }}\right)
$$

is the most probable relaxation time, $T$ thermodynamic temperature and $k_{B}$ the Boltzmann constant [9]. The relaxation processes, described by the Arrhenius equation (2), are characterized by the activation energy $E_{a}$ for jumps over the barrier between two potential minima and typical relaxation frequency of ion hopping $1 / \tau_{0} \approx 10^{13}-10^{14} \mathrm{~s}^{-1}$.

All the glasses we studied using acoustic spectroscopy exhibit the Arrhenius - type relaxation between the temperature of the peak maximum $T_{\text {peak }}$ and the applied frequency $f$ of acoustic waves

$$
f=f_{0} \exp \left(-\frac{E_{a}}{k_{B} T_{\text {peak }}}\right),
$$

where $f_{0}$ is the preexponential factor.

In fact all the investigated relaxation peaks are much broader than Debye peak. It can be interpreted as arising from the existence of a distribution of relaxation times due to random deviations in the local arrangement of the system.

In solid electrolytes the mobile ion concentrations are large and conduction mechanisms are thought to be cooperative. The relaxation phenomena observed in a wide variety of materials exhibit then a power-law type of frequency dependence. The relationship corresponding to the Debye behaviour is expressed in the form [8]

$$
\alpha \approx \frac{1}{T}\left(\frac{(\omega \tau)^{m}}{1+(\omega \tau)^{1+m+n}}\right),
$$

where $m$ and $n$ are power-law exponents, which take values between 0 and 1 . When $m=1$ and $n=0$, equation (4) reduces to the equation for a single Debye-like process. One of the functions which were mainly used to fit mechanical loss data is the double power law (DPL) [10]

$$
\alpha(\omega, T) \propto \frac{1}{(\omega \tau)^{-n}+(\omega \tau)^{n}} .
$$


Using this function, we can fit also the acoustic attenuation spectrum of the lithium phosphate glasses. Applying the method of genetic algorithm [11] with binary representation of the theoretical attenuation DPL function of variables in connection with the visual construction of the acoustic attenuation model we analyzed then the acoustic spectra obtained on copper phosphate glasses.

\section{Experimental results and discussion}

The acoustic spectra are illustrated for investigated sample $\mathrm{BN}$ and measured at various frequencies in Fig. 2. All acoustic measurements indicate one broad attenuation peak at higher temperature, however, for some dependences we can distinguish two separated peaks.

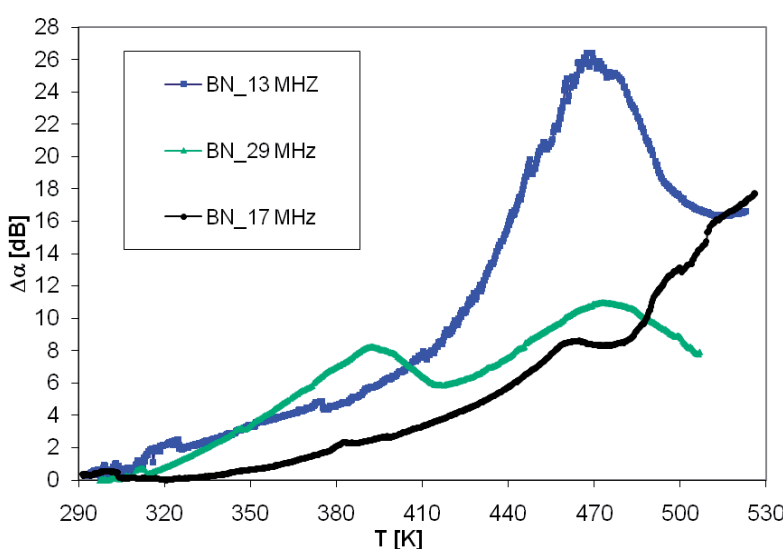

Fig. 2 Comparison of the acoustic attenuation spectra of investigated lithium phosphate glass sample $\mathrm{Li}_{1.22} \mathrm{PO}_{2.80} \mathrm{~N}_{0.21}$ (BN) measured at frequency $f=13,17$ and $29 \mathrm{MHz}$

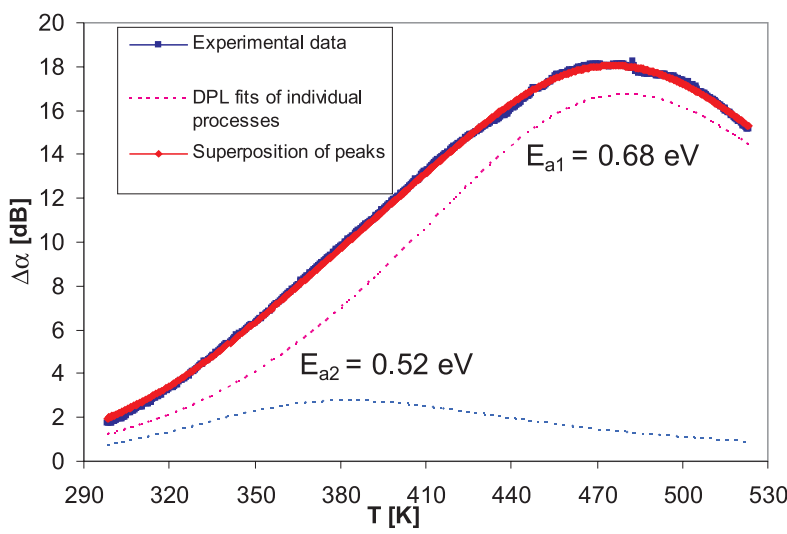

Fig. 3 The acoustic spectrum of the glass sample $\mathrm{Li}_{1.35} \mathrm{PO}_{3.18}(\mathrm{C})$ (full blue line) measured at frequency $f=13 \mathrm{MHz}$ and the DPL fits of the two supposed relaxation processes (dashed line) including their superposition (full red line)
Applying DPL function (5) we simulated acoustic attenuation spectra at constant frequency as a superposition of individual peaks (Figs. 3-5) and determined the values of activation energies (Tab. 1) of the individual relaxation processes connected with ion hopping.

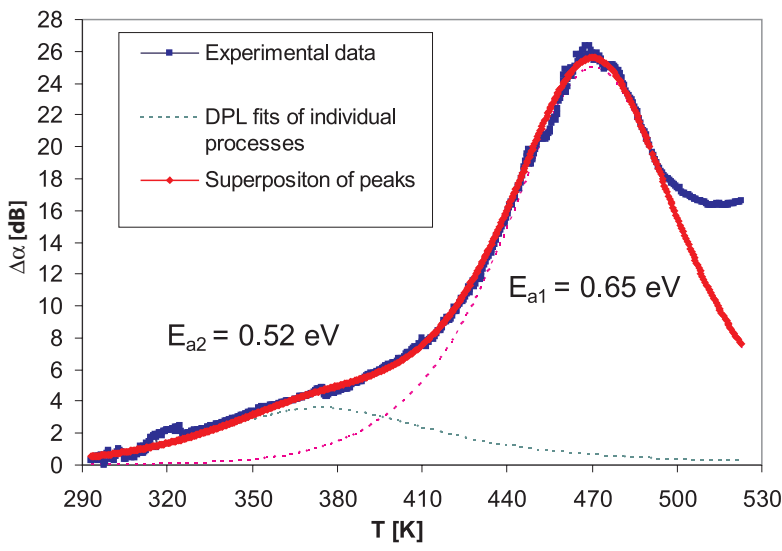

Fig. 4 The acoustic spectrum of the glass sample $\mathrm{Li}_{1.22} \mathrm{PO}_{2.80} \mathrm{~N}_{0.21}$ (BN) (full blue line) measured at frequency $f=13 \mathrm{MHz}$ and the DPL fits of the two supposed relaxation processes (dashed line) including their superposition (full red line)

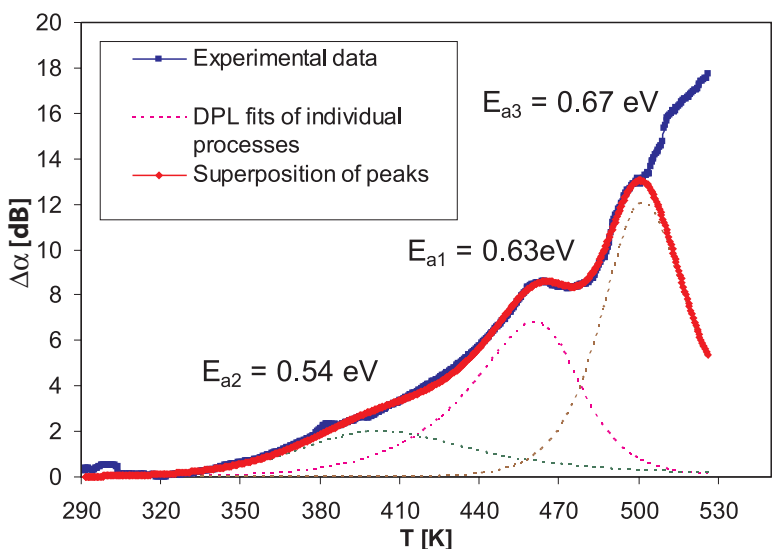

Fig. 5 The acoustic spectrum of glass sample $\mathrm{Li}_{1.22} \mathrm{PO}_{2.80} \mathrm{~N}_{0.21}(\mathrm{BN})$ (full blue line) measured at frequency $f=17 \mathrm{MHz}$ and the DPL fits of the three supposed relaxation processes (dashed line) including their superposition (full red line)

Using the theoretical double power law (DPL) model we tried to fit the whole acoustic attenuation spectra of investigated samples measured at various frequencies as a superposition of two relaxation processes, respectively. The whole temperature dependence of acoustic attenuation cannot be fitted applying only one DPL function in any case. The whole temperature dependence of acoustic attenuation was analyzed then assuming the existence of at least two thermally activated relaxation processes of $\mathrm{Li}^{+}$ions in connection with different kinds of sites. 
There are several categories of relaxation processes connected with temperature peaks of individual processes. Comparing the activation energies obtained from acoustic and electrical measurements (Tab. 1), it seems that essentially the same microscopic processes can be responsible for the electrical and one of the acoustic relaxation processes (the same or very close values of $E_{a 1}$ and $E_{a e}$ ). However, the acoustic spectra are able to detect also other processes compared with electrical spectroscopy.

Activation energies calculated from acoustic

Table 1 and electrical measurements

\begin{tabular}{|c|c|c|c|c|c|}
\hline \multirow{2}{*}{ Glass sample } & \multicolumn{4}{|c|}{ Acoustic Measurements } & $\begin{array}{c}\text { Electrical } \\
\text { measurements }\end{array}$ \\
\cline { 2 - 5 } & $\begin{array}{c}\mathrm{f} \\
{[\mathrm{MHz}]}\end{array}$ & $\begin{array}{c}\mathrm{E}_{\mathrm{a} 1} \\
{[\mathrm{eV}]}\end{array}$ & $\begin{array}{c}\mathrm{E}_{\mathrm{a} 2} \\
{[\mathrm{eV}]}\end{array}$ & $\begin{array}{c}\mathrm{E}_{\mathrm{a} 3} \\
{[\mathrm{eV}]}\end{array}$ & $\begin{array}{c}\mathrm{E}_{\mathrm{ae}} \\
{[\mathrm{eV}]^{*}}\end{array}$ \\
\hline $\begin{array}{c}\mathrm{C} \\
\left(\mathrm{Li}_{1.35} \mathrm{PO}_{3.18}\right)\end{array}$ & 13 & 0.68 & 0.52 & - & 0.68 \\
\hline \multirow{2}{*}{$\begin{array}{c}\mathrm{BN} \\
\left(\mathrm{Li}_{1.22} \mathrm{PO}_{2.80} \mathrm{~N}_{0.21}\right)\end{array}$} & 13 & 0.65 & 0.52 & - & \multirow{2}{*}{0.64} \\
\cline { 2 - 5 } & 17 & 0.63 & 0.54 & 0.67 & \multirow{2}{*}{0.67} \\
\hline
\end{tabular}

*Data were used from [2]

The results from FTIR spectra of the $\mathrm{LiPO}_{3}$ glasses [1] show characteristic peaks corresponding to the different vibration modes of the $\mathrm{PO}_{4}$ tetrahedra $\left(v\left(\mathrm{PO}_{4}\right)^{3-}{ }_{\text {sym,asym }}\right)$ as well as those of the $\mathrm{P}-\mathrm{O}-\mathrm{P}$ bonds $\left(v(\mathrm{P}-\mathrm{O}-\mathrm{P})_{\text {sym, asym }}\right.$ and $\left.\delta(\mathrm{P}-\mathrm{O}-\mathrm{P})\right)$ and the $\mathrm{PO}_{2}{ }^{-}$ groups $\left(v\left(\mathrm{PO}_{2}\right)^{-}\right.$sym,asym $)$. Using Nuclear Magnetic Resonance it was found [12] that the oxide glass marked $\mathrm{C}$ (nitrogen-free) is composed of $\mathrm{PO}_{4}$ groups of $\mathrm{Q}^{2}$ - type (metaphosphate composition with two bridging oxygen atoms bonded to neighbouring phosphorous atoms, cyclic anions of exact $\left.\left(\mathrm{PO}_{3}{ }^{-}\right)_{\mathrm{n}}\right)$ and $\mathrm{Q}^{1}$ structural units (pyrophosphate composition with one bridging oxygen atom bonded to neighbouring phosphorous atom, $\mathrm{P}_{2} \mathrm{O}_{7}{ }^{4-}$ groups). Furthermore, oxynitride glass marked $\mathrm{BN}$ presents other groups: $\mathrm{PO}_{3} \mathrm{~N}$ and $\mathrm{PO}_{2} \mathrm{~N}_{2}$, which are results of the nitrogen/oxygen substitution [13]. The two activated processes found from modelling of acoustic spectra might be related to different structural motifs: Lithium ions bonded to non-bridging oxygen atoms present in $\mathrm{Q}^{2}$ phosphate units or $\mathrm{Q}^{1}$ pyrophosphate ones. Apart from that, it would be necessary to take into account that non-bridging oxygen atoms belonging to $\mathrm{PO}_{3} \mathrm{~N}$ and $\mathrm{PO}_{2} \mathrm{~N}_{2}$ oxynitride tetrahedral units formed during nitridation might also contribute with additional effects, e.g. a third process.
From acoustic measurements we found lower activation energies for the sample $\mathrm{BN}$ in comparison with the sample $\mathrm{C}$. It was found that nitrogen affected the electrical conductivity according to the structural modifications of the glass network induced by nitrogen [2]. Some authors [14] supposed that the decrease in the electrostatic energy when $\mathrm{P}-\mathrm{O}$ bonds are replaced by more covalent $\mathrm{P}-\mathrm{N}$ bonds may cause the decrease in the electrostatic activation energy and increase in ionic conductivity. The effect of nitrogen on conductivity can be explained by higher cross-linking density introduced by nitrogen atoms, which should facilitate the lithium transfer between phosphate chains. The higher amount of non-bridging oxygen atoms generated by nitridation could increase the number of hopping positions for $\mathrm{Li}^{+}$ions creating conduction paths with lower activation energy and the rising of the electrical conductivity as a consequence of this [2].

\section{Conclusion}

The experimental investigation of the lithium phosphate and oxynitride phosphate glasses proved that acoustical spectroscopy can be a very useful technique for the study of relaxation and transport mechanisms in ion conductive glasses. Using the theoretical model of Double Power Law function for the simulation of acoustic spectra we can better determine and describe the relaxation processes and transport mechanism of mobile ions. Several different kinds of sites responsible for ionic hopping motion were discovered and described. The comparison of activation energies obtained from acoustic and electrical measurements showed that the same microscopic processes can be responsible for the acoustic and electrical relaxation processes, however the acoustic spectroscopy can detect also some minor processes. It was found that the activation energy connected with main attenuation peak decreases with increasing content of nitrogen. Nitridation increases the amount of non-bridging oxygen atoms and cross-linking density which increases the electrical conductivity of the oxynitride phosphate glasses connected with decrease of the activation energy.

\section{Acknowledgments}

The authors would like to thank to Mr. F. Cernobila for technical assistance. The authors wish to thank for the support to the $R \& D$ operational program "Centre of excellence of power electronics systems and materials" for their components. The project is funded by the European Community, ERDF - European regional development fund.

\section{References}

[1] MOREAU, F., DURAN, A., MUNOZ, F.: Journal of the European Ceramic Society 29 (2009) 1895

[2] MUNOZ, F., DURAN, A., PASCUAL, L., MONTAGNE, L., REVEL B., RODRIGUES, A. C. M.: Solid State Ionics 179 (2008) 574

[3] YU, X., BATES, J. B., JELLISON JR., G. E., HART, F. X.: J. Electrochem. Soc. 144 (2) (1997) 524

[4] ROLING, B., HAPPE, A., INGRAM, M. D., FUNKE, K.: J. Phys. Chem. B 103 (1999) 4122 


\section{COMMNICOIIONS}

[5] CHARNAZA, E. V., BORISOV, B. F., KULESHOV, A. A.: Proc. World Congress on Ultrasonics, Berlin (1995) 483

[6] FUNKE, K.: Sol. State Ionics 94 (1997) 27

[7] BURY, P., HOCKICKO, P., JURECKA, S., JAMNICKY, M.: Physica Status Solidi (c) 11 (2004) 2888

[8] ALMOND, D. P., WEST, A. R.: Solid State Ionics 26 (1988) 265

[9] CARINI, G., CUTRONI, M., FEDERICO, M., GALLI, G., TTIPODO, G.: Physical Review B 30 (1984) 7219

[10] BURY, P., HOCKICKO, P., JAMNICKY, M.: Advanced Materials Research 39-40 (2008) 111

[11] JURECKA, S., JURECKOVA, M., MULlEROVA, J.: Acta Physica Slovaca 53 (2003) 215

[12] BROW, R. K.: J. Non-Cryst. Solids 263\&264 (2000) 1

[13] MUNOZ, F., PASCUAL, L., DURAN, A., MONTAGNE, L., PALAVIT, G., BERJOAN, R., MARCHAND, R.: J. Non-Cryst. Solids 324 (2003) 142

[14] WANG, B., KWAK, B. S., SALES, B. C., BATES, J. B.: J. Non-Cryst. Solids 183 (1995), 297. 